\title{
Observables suitable for restricting the fidelity to multipartite maximally entangled states
}

\author{
Koji Nagata, Masato Koashi, and Nobuyuki Imoto \\ CREST Research Team for Interacting Carrier Electronics, School of Advanced Sciences, \\ The Graduate University for Advanced Studies (SOKEN), Hayama, Kanagawa, 240-0193, Japan
}

(Dated: September 5, 2018)

\begin{abstract}
We present a class of observables which are suitable for determining the fidelity of a state to the multipartite Greenberger-Horne-Zeilinger (GHZ) state. Given an expectation value of an observable belonging to the class, we give a simple formula that gives a lower bound and an upper bound for the fidelity. Applying the formula to the GHZ-state preparation experiment by Pan et al. [Nature (London) 403, 515 (2000)], we show that the observed state lies outside of the class of biseparable mixed three-qubit states. We also show that for this class of operators, adopting the principle of minimum variance [Phys. Rev. A 60, 4338 (1999)] in the state estimation always results in the state with the minimum fidelity.

PACS numbers: 03.67.-a, 03.65.Fd, 03.65.Ud, 03.65.Wj
\end{abstract}

\section{INTRODUCTION}

Recently, importance of entangled states of multipartite system has been realized not only as a fundamental concept of quantum mechanics [1] but also as an essential resource for quantum information processing [2]. Up to now, there have already been several experimental reports on 3- and 4-particle entangled states [3, 4, 5]. One of the important measures to analyze how close the produced quantum state is to the desired maximally entangled state is the fidelity $[\underline{6}$, i.e., the overlap with the desired entangled state. For bipartite systems and multipartite systems, the fidelity is used as a criterion for nonseparability and distillability of the so-called Werner state or Werner-type state, i.e., a maximally entangled state mixed with the completely depolarized state 7 ]. In the classification of mixed three-qubit states [8], it was shown that one of tripartite witnesses can be used to detect a state that does not belong to the biseparable class. This witness is given by

$$
\mathcal{W}=\frac{1}{2} \mathbb{1}-P_{\mathrm{GHZ}},
$$

where $P_{\mathrm{GHZ}}$ is the projector onto a GHZ state. When $\operatorname{Tr}[\mathcal{W} \rho]<0$, the state $\rho$ lies outside of the biseparable class and has genuinely tripartite entanglement. Since $\operatorname{Tr}[\mathcal{W} \rho]$ is written as $1 / 2-f$ using the fidelity $f$ to the GHZ state, the fidelity is useful to determine to which class a state belongs $[9]$.

As can be seen from these examples, it is important to determine the fidelity $f$ of experimentally produced states from the observed data. Systematic arguments about the possible fidelity values allowed by experimental data for multipartite system will be helpful to the experimental realization of various applications in quantum information processing. The main purpose of this paper is to give a formula for the possible range of the fidelity value in the simplest case, i.e., the case where an expectation value of a single operator is given as experimental data. We present a class of observables which are suitable for determining the range of the fidelity of a state to the $n$-partite GHZ state $\left|\Phi_{n}\right\rangle$. The class is determined through the expansion of the projection operator $\left|\Phi_{n}\right\rangle\left\langle\Phi_{n}\right|$ into the sum of direct products of Pauli operators for each party. Given an expectation value of an observable belonging to the class, the formula gives a lower bound and an upper bound for the fidelity. As an example, we analyze the GHZ-state preparation experiment by Pan et al. [4] and show that the fidelity to the GHZ state is larger than 0.71. This indicates that the observed state does not belong to the biseparable class and has genuinely tripartite entanglement.

In addition to the argument of what kind of states are possible under the constraints of experimental data, there also is a problem of determining which state is most likely under the constraints. Such problems of estimation for bipartite system has been discussed along the maximum entropy principle 10]. Application of only this principle sometimes leads to an estimated state that possesses stronger entanglement than the minimum entanglement that is compatible with the measured data. Based on the additional assumption that the realization of a stronger entanglement is less realistic, Horodecki et al. introduced a new constraint, i.e., minimization of entanglement 11] in applying the maximum entropy principle. They thus obtained an estimated state that has the minimum entanglement. Rajagopal derived the same state with a different assumption together with the the maximum entropy principle, i.e., to minimize the variance of a Bell operator 12]. Since then, much attention has been paid [13] to this problem. Here we will show that, in multipartite systems, applying the minimum variance principle to the operators belonging to the above class gives the states with the minimum fidelity that is allowed by the constraints. This is a generalization of Rajagopal's results to multipartite systems, and reveals why and in what cases the minimum variance leads to small entanglement.

This paper is organized as follows. In Sec. III we take a GHZ state as the state of interest and present the class 
of operators by decomposing the projector into the sum of operators forming a commutative group. In Sec. III we derive a simple formula that gives a lower bound and an upper bound for the fidelity. In Sec. IV we apply the formula to analyze the GHZ-state preparation experiment by Pan et al. [4], and show that the fidelity to the GHZ state is larger than 0.71. Sec. $\mathrm{V}$ deals with the state estimation problem based on the minimum variance principle. Sec. VI concludes this paper.

\section{DECOMPOSITION OF PROJECTOR AND CLASS OF OBSERVABLES}

In order to discuss the fidelity, we have to specify one state of interest. We have referred to the state as the desired state. we take $n$-partite GHZ state $\left|\Phi_{n}\right\rangle$ as the desired state, which is defined as

$$
\left|\Phi_{n}\right\rangle:=\frac{1}{\sqrt{2}}\left(\left|+_{1} ;+_{2} ; \cdots ;+_{n}\right\rangle+\left|-_{1} ;-_{2} ; \cdots ;-{ }_{n}\right\rangle\right) .
$$

In the following discussion, we consider the fidelity of the state $\rho$ to $\left|\Phi_{n}\right\rangle$, i.e., $f:=\operatorname{Tr}\left[\rho\left|\Phi_{n}\right\rangle\left\langle\Phi_{n}\right|\right]$. The projection operator $\left|\Phi_{n}\right\rangle\left\langle\Phi_{n}\right|$ can be expanded as

$$
\begin{aligned}
\left|\Phi_{n}\right\rangle\left\langle\Phi_{n}\right|= & \frac{1}{2^{n+1}}\left(\prod_{j=1}^{n}\left(\sigma_{x}^{j}+i \sigma_{y}^{j}\right)+\prod_{j=1}^{n}\left(\sigma_{x}^{j}-i \sigma_{y}^{j}\right)\right. \\
& \left.+\prod_{j=1}^{n}\left(I^{j}+\sigma_{z}^{j}\right)+\prod_{j=1}^{n}\left(I^{j}-\sigma_{z}^{j}\right)\right) \\
= & \left(1 / 2^{n}\right)\left(O_{0}+O_{1}+\cdots+O_{2^{n}-1}\right)
\end{aligned}
$$

where $O_{p}$ is defined by

$$
O_{p}:=\prod_{j=1}^{n}\left(\sigma_{x}^{j}\right)^{b_{0}}\left(\sigma_{z}^{j}\right)^{b_{j}}
$$

where the $n$-bit sequence $b_{0} b_{1} \cdots b_{n-1}$ is the binary representation of $p, b_{n}=\sum_{j=1}^{n-1} b_{j}$, since the terms with odd parity for $b_{1} \cdots b_{n}$ vanish in the above expansion. The superscript $j$ of the Pauli operators denotes particle $j$. It is easy to see that $O_{p} O_{q}=O_{p \oplus q}$, where $p \oplus q$ is the bitwise XOR of $p$ and $q$. Hence the set of $2^{n}$ operators $\left\{O_{p}\right\}$ forms a commutative group isomorphic to $\left(Z_{2}\right)^{n}$. We denote this commutative group as $\Lambda_{n}$. The operator $\mathrm{O}_{0}$ is the identity operator for the $2^{n}$-dimensional space, and the other operators $O_{1}, \cdots, O_{2}{ }^{n-1}$ have two eigenvalues, \pm 1 . All elements of $\Lambda_{n}$ take $\left|\Phi_{n}\right\rangle$ as an eigenstate with eigenvalue 1 .

For $n=2$, the above expansion is explicitly written as follows,

$$
\left|\Phi_{2}\right\rangle\left\langle\Phi_{2}\right|=(1 / 4)\left(I^{1} I^{2}+\sigma_{z}^{1} \sigma_{z}^{2}+i \sigma_{y}^{1} i \sigma_{y}^{2}+\sigma_{x}^{1} \sigma_{x}^{2}\right),(5)
$$

where $I$ represents the identity operator for the 2dimensional space. For $n=3$, it is written as

$$
\begin{aligned}
& \left|\Phi_{3}\right\rangle\left\langle\Phi_{3}\right|=(1 / 8)\left(\mathcal{O}_{I I I}+\mathcal{O}_{I z z}+\mathcal{O}_{z I z}+\mathcal{O}_{z z I}\right. \\
& \left.\quad+\mathcal{O}_{x x x}+\mathcal{O}_{x y y}+\mathcal{O}_{y x y}+\mathcal{O}_{y y x}\right)
\end{aligned}
$$

where we have used the simplified notations as $\mathcal{O}_{I I I}:=$ $I^{1} I^{2} I^{3}, \quad \mathcal{O}_{I z z}:=I^{1} \sigma_{z}^{2} \sigma_{z}^{3}, \quad \mathcal{O}_{z I z}:=\sigma_{z}^{1} I^{2} \sigma_{z}^{3}, \quad \mathcal{O}_{z z I}:=$ $\sigma_{z}^{1} \sigma_{z}^{2} I^{3}, \mathcal{O}_{x x x}:=\sigma_{x}^{1} \sigma_{x}^{2} \sigma_{x}^{3}, \mathcal{O}_{x y y}:=\sigma_{x}^{1} i \sigma_{y}^{2} i \sigma_{y}^{3}, \mathcal{O}_{y x y}:=$ $i \sigma_{y}^{1} \sigma_{x}^{2} i \sigma_{y}^{3}$, and $\mathcal{O}_{y y x}:=i \sigma_{y}^{1} i \sigma_{y}^{2} \sigma_{x}^{3}$.

Now let us consider the problem of determining the fidelity by measuring the expectation value of an observable. The most direct approach is, of course, to measure $\left\langle\mid \Phi_{n}\right\rangle\left\langle\Phi_{n} \mid\right\rangle$, which will be done by conducting $2^{n}-1$ different correlation measurements to determine $\left\langle O_{j}\right\rangle\left(j=1,2, \cdots, 2^{n}-1\right)$. Our interest here is how we can deduce the information about the fidelity from observables that can be measured by much smaller number of correlation measurements. In what follows, we give a formula to derive an inequality for the fidelity from an expectation value of an observable belonging to the class $\mathcal{C}_{n}$ defined as follows.

Class $\mathcal{C}_{n}$ : An observable $\mathcal{A}$ belongs to $\mathcal{C}_{n}$ if and only if $\mathcal{A}$ is a linear combination of operators $Q_{1}, Q_{2}, \cdots, Q_{m} \in \Lambda_{n}$ with positive coefficients, and the set $\left\{Q_{1}, Q_{2}, \cdots, Q_{m}\right\}$ forms a system of generators for $\Lambda_{n}$.

The class is determined through $\Lambda_{n}$, and hence determined by the desired state $\left|\Phi_{n}\right\rangle$.

The minimum cardinal number of systems of generators for $\Lambda_{n}$ is $n$. To see this, suppose there are only $l(<n)$ generators. In this case, however, they can generate at most $\sum_{i=0}^{l} C_{i}=2^{l}\left(<2^{n}\right)$ kinds of elements of $\Lambda_{n}$ due to the property of the Pauli matrices. An example of a system of generators for $\Lambda_{n}$ is

$$
\{O_{x, x, \cdots, x}, \overbrace{O_{z, I, I, \cdots, z}, O_{I, z, I, I, \cdots, z}, \cdots, O_{I, I, \cdots, z, z}}^{n-1}\},
$$

where $O_{x, x, \cdots, x}:=\sigma_{r}^{1} \sigma_{x}^{2} \cdots \sigma_{x}^{n}, \quad O_{I, z, I, \cdots, z}:=$ $I^{1} \sigma_{z}^{2} I^{3} \cdots \sigma_{z}^{n}$, and so on 14$]$. This system of generators for $\Lambda_{n}$, indeed, generates all $2^{n}$ elements of $\Lambda_{n}$ with the help of Eq. (41).

As for $\Lambda_{2}$, examples of a system of generators are $\left\{\sigma_{x}^{1} \sigma_{x}^{2}, \sigma_{z}^{1} \sigma_{z}^{2}\right\},\left\{\sigma_{x}^{1} \sigma_{x}^{2}, \sigma_{z}^{1} \sigma_{z}^{2}, i \sigma_{y}^{1} i \sigma_{y}^{2}\right\},\left\{I, \sigma_{x}^{1} \sigma_{x}^{2}, i \sigma_{y}^{1} i \sigma_{y}^{2}\right\}$ and so on. As for $\Lambda_{3}$, examples of a system of generators are $\left\{\mathcal{O}_{x y y}, \mathcal{O}_{y x y}, \mathcal{O}_{y y x}\right\},\left\{\mathcal{O}_{x y y}, \mathcal{O}_{y x y}, \mathcal{O}_{I z z}\right\},\left\{\mathcal{O}_{x x x}\right.$, $\left.\mathcal{O}_{x y y}, \mathcal{O}_{y x y}, \mathcal{O}_{y y x}\right\}$ and so on.

\section{INEQUALITY FOR FIDELITY}

In this section, we derive an inequality under the condition that the expectation value of an operator $\mathcal{A} \in \mathcal{C}_{n}$ is specified. First, we will show that if the given expectation value is the maximum value, the state must be $\left|\Phi_{n}\right\rangle$. For that, we use the following lemma.

Lemma. Let $X, Y$ be operators taking eigenvalues \pm 1 , and $[X, Y]=0$. Then,

$$
1-|\langle X\rangle-\langle Y\rangle| \geq\langle X Y\rangle \geq\langle X\rangle+\langle Y\rangle-1
$$

This is directly proven by inequalities:

$$
\langle(1-X)(1-Y)\rangle \geq 0 \quad \text { and } \quad\langle(1 \pm X)(1 \mp Y)\rangle \geq 0
$$


In the following, we consider $\mathcal{A}=\alpha_{1} Q_{1}+\alpha_{2} Q_{2}+\cdots+$ $\alpha_{m} Q_{m}\left(2^{n} \geq m \geq n, \alpha_{i}>0, \forall i\right)$. We assume that $\mathcal{A} \in \mathcal{C}_{n}$. We assume that the maximum expectation value $\langle\mathcal{A}\rangle=M$ is given, where $M:=\sum_{i=1}^{m} \alpha_{i}$ is the maximum eigenvalue of $\mathcal{A}$. Because all coefficients $\alpha_{i}$ are positive, $\langle\mathcal{A}\rangle=M$ implies $\left\langle Q_{1}\right\rangle=\left\langle Q_{2}\right\rangle=\cdots=\left\langle Q_{m}\right\rangle=1$. Since $Q_{j}^{2}=I$ for any $j$ and the set $\left\{Q_{1}, Q_{2}, \cdots, Q_{m}\right\}$ forms a system of generators for $\Lambda_{n}$, any element $Q$ of $\Lambda_{n}$ can be written as

$$
Q=Q_{\beta_{1}} Q_{\beta_{2}} \cdots Q_{\beta_{l}}
$$

with $1 \leq \beta_{1}<\beta_{2}<\cdots<\beta_{l} \leq m$. We show that $\langle Q\rangle=1$ for all $l$ as follows. When $l=1$, then $\langle Q\rangle=1$ holds. Suppose $\langle Q\rangle=1$ holds when $l=k$, i.e., $\left\langle\prod_{i=1}^{k} Q_{\beta_{i}}\right\rangle=1$. Then with the help of the lemma, $\left\langle\prod_{i=1}^{k+1} Q_{\beta_{i}}\right\rangle=1$ holds. Let $X$ be $\prod_{i=1}^{k} Q_{\beta_{i}}$ and $Y$ be $Q_{\beta_{k+1}}$, respectively. The lemma leads that $1 \leq\left\langle\prod_{i=1}^{k+1} Q_{\beta_{i}}\right\rangle \leq 1$. Hence $\left\langle Q_{1}\right\rangle=$ $\left\langle Q_{2}\right\rangle=\cdots=\left\langle Q_{m}\right\rangle=1$ means the expectation values of all elements of $\Lambda_{n}$ are one. This means that the fidelity is 1 with the help of Eq. (3). Therefore we obtain the following:

Proposition 1: Let $M$ be the largest eigenvalue of $\mathcal{A} \in$ $\mathcal{C}_{n}$. If $\operatorname{Tr}[\rho \mathcal{A}]=M$, then $\rho=\left|\Phi_{n}\right\rangle\left\langle\Phi_{n}\right|$.

This implies that the largest eigenvalue $M$ is not degenerate. This is crucial point in deriving an inequality for the fidelity. Let us write the eigenvalues of $\mathcal{A}$ as $M, r_{2}, r_{3}, \cdots$, and $r_{s}\left(s \leq 2^{n}\right)$, where $M>r_{2}>r_{3}>$ $\cdots>r_{s} \geq-M$. In this notation, when some eigenvalues of $\mathcal{A}$ are degenerate, then $s<2^{n}$ holds, and when all the eigenvalues of $\mathcal{A}$ are not degenerate, then $s=2^{n}$ holds. Since proposition 1 implies that $\left|\Phi_{n}\right\rangle$ is the only eigenstate for the largest eigenvalue $M$, we can generally expand $\langle\mathcal{A}\rangle:=\operatorname{Tr}[\rho \mathcal{A}]$ as

$$
\langle\mathcal{A}\rangle=M f+\sum_{i=2}^{s} q_{i} r_{i}
$$

where $f=\operatorname{Tr}\left[\rho\left|\Phi_{n}\right\rangle\left\langle\Phi_{n}\right|\right]$ is the fidelity to $\left|\Phi_{n}\right\rangle$, and $q_{i}(\geq 0)$ satisfy $f+\sum_{i=2}^{s} q_{i}=1$. Using this relation to eliminate $q_{2}$, we have

$$
\begin{aligned}
\langle\mathcal{A}\rangle & =M f+\left(1-f-\sum_{i=3}^{s} q_{i}\right) r_{2}+\sum_{i=3}^{s} q_{i} r_{i} \\
& =\left(M-r_{2}\right) f+r_{2}-\sum_{i=3}^{s} q_{i}\left(r_{2}-r_{i}\right),
\end{aligned}
$$

We thus obtain

$$
\frac{\langle\mathcal{A}\rangle-r_{2}}{M-r_{2}} \leq f
$$

The equality of the relation (13) holds when $\sum_{i=3}^{s} q_{i}=0$.

We can also derive an inequality that gives an upper bound of the fidelity by eliminating $q_{s}$, namely,

$$
\begin{aligned}
\langle\mathcal{A}\rangle & =M f+\sum_{i=2}^{s-1} q_{i} r_{i}+\left(1-f-\sum_{i=2}^{s-1} q_{i}\right) r_{s} \\
& =\left(M-r_{s}\right) f+\sum_{i=2}^{s-1} q_{i}\left(r_{i}-r_{s}\right)+r_{s},
\end{aligned}
$$

and

$$
\frac{\langle\mathcal{A}\rangle-r_{s}}{M-r_{s}} \geq f
$$

The equality of the relation (15) holds when $\sum_{i=2}^{s-1} q_{i}=0$. We therefore obtain the following proposition.

Proposition 2: Let $M, r_{2}$, and $r_{s}$ be the largest, the second-largest, and the smallest eigenvalue of $\mathcal{A} \in \mathcal{C}_{n}$, respectively. When $\langle\mathcal{A}\rangle:=\operatorname{Tr}[\rho \mathcal{A}]$ is given, the fidelity $f:=\operatorname{Tr}\left[\rho\left|\Phi_{n}\right\rangle\left\langle\Phi_{n}\right|\right]$ is bounded as

$$
\frac{\langle\mathcal{A}\rangle-r_{2}}{M-r_{2}} \leq f \leq \frac{\langle\mathcal{A}\rangle-r_{s}}{M-r_{s}} .
$$

\section{APPLICATION TO EXPERIMENTAL DATA}

We analyze the experimental data by Pan et al. [4]. In this experiment they obtained four expectation values of three-photon polarization correlations,

$$
\begin{aligned}
& \langle x y y\rangle \simeq\langle y x y\rangle \simeq\langle y y x\rangle \simeq 0.70, \\
& \langle x x x\rangle \simeq 0.74 .
\end{aligned}
$$

These experimental data is obtained by the post selection, i.e., picking up only the events with each of the three detectors registering a photocount. If the detectors used in the experiments were ideal ones, the post-selected state would be contained in a $2^{3}$-dimensional subspace, which can be identified with a tripartite system of three qubits. Then the above observed values could be considered to give the expectation values $\left\langle\mathcal{O}_{x y y}\right\rangle,\left\langle\mathcal{O}_{y x y}\right\rangle$, $\left\langle\mathcal{O}_{y y x}\right\rangle$, and $\left\langle\mathcal{O}_{x x x}\right\rangle$. In the real experiment, however, the detectors are not ideal, namely, they cannot distinguish a single photon from more than one photons and they have a limited quantum efficiency and dark counting. Due to these imperfection together with the nonideal photon source, the post-selected state also contains contributions outside of the $2^{3}$-dimensional subspace, in which two photons or no photons enter the same detector. However, the superfluous contributions can be neglected as compared to the statistical uncertainty (a few \%) of the observed values as follows $[3]$. The contribution of nophoton events are due to dark counting, but the rate of the dark counts is low enough [3] to be able to neglect the effect. The contribution of more than one photon entering a detector passes the post selection only if another detector have a dark count, or more than two photon pairs are created in the parametric downconversion. The former case is negligible due to the low dark count rate, 
and the latter is also negligible since the probability per pulse to create $n$-photon pairs is of the order of about $10^{-4 n}$. We can thus assume that the post-selected state is related to polarization of three photons and approximately supports $2^{3}$-dimensional Hilbert space. Hence we obtain

$$
\begin{aligned}
& \left\langle\mathcal{O}_{x y y}\right\rangle \simeq\left\langle\mathcal{O}_{y x y}\right\rangle \simeq\left\langle\mathcal{O}_{y y x}\right\rangle \simeq 0.70 \\
& \left\langle\mathcal{O}_{x x x}\right\rangle \simeq 0.74
\end{aligned}
$$

The limited quality of the polarization optics just before the detectors may make the visibility lower, which will make the estimated fidelity smaller. Hence we use these experimental expectation values for restricting the fidelity from below.

Clearly, each observable of (18) does not belong to $\mathcal{C}_{3}$. Therefore we take the summation of these expectation values. We then obtain

$$
\langle\mathcal{A}\rangle \simeq 2.84
$$

where $\mathcal{A}=\mathcal{O}_{x y y}+\mathcal{O}_{y x y}+\mathcal{O}_{y y x}+\mathcal{O}_{x x x}$. Apparently, $\mathcal{A} \in$ $\mathcal{C}_{3}$ holds, and eigenvalues of $\mathcal{A}$ are 4 , 0 , and -4 , where 0 is a degenerate eigenvalue. With the help of Eq. (16), where the parameters are set to $M=4$ and $r_{2}=0$, we can state that the observed state in this experiment has the fidelity to a GHZ state larger than or equal to $0.71[15$. Because the value is lager than $1 / 2$, with the help of Eq. (1), these experimental data, indeed, ensure the observed state does not belong to the biseparable class and has genuinely tripartite entanglement.

\section{RELATION BETWEEN VARIANCE AND FIDELITY}

In this section, we show that if we apply the minimum variance principle for estimating the states from an experimentally obtained expectation value of an operator belonging to $\mathcal{C}_{n}$, the estimated fidelity becomes the minimum value that is allowed by the constraints. This is a generalization of Rajagopal's results [12] to multipartite systems.

Let us consider Rajagopal's case, i.e., bipartite system in which the variance of Bell-CHSH operator $B=$ $\sqrt{2}\left(\sigma_{x}^{1} \sigma_{x}^{2}+\sigma_{z}^{1} \sigma_{z}^{2}\right)$ is made minimal.

Suppose that an expectation value $\langle\mathcal{A}\rangle$ is given, where

$$
\begin{array}{ll}
\text { Case } 1 & \mathcal{A}=\sigma_{x}^{1} \sigma_{x}^{2}, \\
\text { Case } 2 & \mathcal{A}=\sigma_{x}^{1} \sigma_{x}^{2}+\sigma_{z}^{1} \sigma_{z}^{2} .
\end{array}
$$

Note that, for Case 1 , the operator $\mathcal{A}$ does not belong to $\mathcal{C}_{2}$, whereas, for Case 2 , the operator $\mathcal{A}$ belongs to $\mathcal{C}_{2}$, and $\mathcal{A}=B / \sqrt{2}$.

For Case 2, combining the results by Refs. 11] and [12], it is shown that if variance $\left\langle(\Delta \mathcal{A})^{2}\right\rangle$ is made minimal, the calculated fidelity to $\left|\Phi_{2}\right\rangle$ takes minimal when the expectation value $\langle\mathcal{A}\rangle(\geq 0)$ is given. In this way, the minimum entangled state was derived from Jaynes principle. For Case 1, the given $\langle\mathcal{A}\rangle$ determines a range $0 \leq f \leq \frac{\langle\mathcal{A}\rangle+1}{2}$ for the possible value of $f$, and the minimization condition for $\left\langle(\Delta \mathcal{A})^{2}\right\rangle$ puts no further condition on this range. The allowed fidelity value is thus unsettled and can be any value in the region, except for the case that $\langle\mathcal{A}\rangle=-1(\rightarrow f=0)$. (Remember $-1 \leq\langle\mathcal{A}\rangle \leq 1$ for Case 1). This also means that, for Case 1, we cannot derive minimum fidelity (i.e., zero) from the minimum variance principle.

Next we consider several examples for tripartite system. There are eight GHZ states, which are written as

$$
\begin{aligned}
& \left|\psi_{1(8)}\right\rangle:=(1 / \sqrt{2})\left(\left|+{ }_{1} ;+_{2} ;+_{3}\right\rangle \pm\left|-_{1} ;-_{2} ;-{ }_{3}\right\rangle\right), \\
& \left|\psi_{2(7)}\right\rangle:=(1 / \sqrt{2})\left(\left|--_{1} ;+_{2} ;+_{3}\right\rangle \pm\left|+_{1} ;-_{2} ;-{ }_{3}\right\rangle\right), \\
& \left|\psi_{3(6)}\right\rangle:=(1 / \sqrt{2})\left(\left|++_{1} ;-_{2} ;+_{3}\right\rangle \pm\left|-_{1} ;+_{2} ;-{ }_{3}\right\rangle\right), \\
& \left|\psi_{4(5)}\right\rangle:=(1 / \sqrt{2})\left(\left|++_{1} ;+_{2} ;-{ }_{3}\right\rangle \pm\left|-_{1} ;-_{2} ;+_{3}\right\rangle\right) .
\end{aligned}
$$

The eight probabilities of observation of these GHZ states for the state $\rho$ are defined as

$$
p_{i}:=\left\langle\psi_{i}|\rho| \psi_{i}\right\rangle, \quad(i=1,2, \cdots, 8) .
$$

We can see that $\left|\psi_{1}\right\rangle$ is equal to $\left|\Phi_{3}\right\rangle$. The fidelity $f$ is then identical to $p_{1}$.

Suppose that an expectation value $\langle\mathcal{A}\rangle$ is given, where we consider

$$
\begin{array}{ll}
\text { Case } 1 & \mathcal{A}=\mathcal{O}_{x y y}+\mathcal{O}_{y x y}, \\
\text { Case } 2 & \mathcal{A}=\mathcal{O}_{x y y}+\mathcal{O}_{y x y}+\mathcal{O}_{z z I}, \\
\text { Case } 3 & \mathcal{A}=\mathcal{O}_{x y y}+\mathcal{O}_{y x y}+\mathcal{O}_{y y x}
\end{array}
$$

Note that, for Case 1 and 2, the operator $\mathcal{A}$ does not belong to $\mathcal{C}_{3}$, whereas, for Case 3 , the operator $\mathcal{A}$ belongs to $\mathcal{C}_{3}$.

For Case 3, when the variance $\left\langle(\Delta \mathcal{A})^{2}\right\rangle$ is made minimal, we see, later, that we can derive the minimum fidelity. For Case 1 and 2, however, the minimum variance principle does not work completely. In Case 1 and 2 , the given $\langle\mathcal{A}\rangle$ determines range $0 \leq f \leq \frac{\langle\mathcal{A}\rangle+2}{4}$ and $0 \leq f \leq \frac{\langle\mathcal{A}\rangle+1}{4}$, respectively for the possible value of $f$. For Case 2, the minimization condition for $\left\langle(\Delta \mathcal{A})^{2}\right\rangle$ puts no further condition on this range 16]. For Case 1 , the minimization of $\left\langle(\Delta \mathcal{A})^{2}\right\rangle$ makes $f_{\max }$ smaller, i.e., the allowed fidelity value is 0 for $-2 \leq\langle\mathcal{A}\rangle \leq 0$ and $0 \leq f \leq \frac{\langle\mathcal{A}\rangle}{2}$ for $0<\langle\mathcal{A}\rangle \leq 2$. (Remember $-2 \leq\langle\mathcal{A}\rangle \leq 2$ for Case 1). This means that, for Case 2, we cannot derive the minimum fidelity (i.e., zero) from the minimum variance principle and for Case 1 , we cannot derive the minimum fidelity for $0<\langle\mathcal{A}\rangle \leq 2$.

Now we calculate the fidelity for Case 3 from the minimum variance principle. If we write operator $\mathcal{A}$ in the matrix form using the GHZ basis, the diagonal elements become $3,-1,-1,-1,1,1,1$, and -3 , and no offdiagonal element appears. This means that the measured value for $\mathcal{A}$ can take four values $3,-1,1$, and -3 , where -1 and 1 are degenerate eigenvalues. Using notations 
$p_{\alpha}:=p_{2}+p_{3}+p_{4}, p_{\beta}:=p_{5}+p_{6}+p_{7}$, and the relation $f=p_{1}$, the probability that the measured value for $\mathcal{A}$ takes $3,1,-1$, or -3 is expressed as $\left\{f, p_{\beta}, p_{\alpha}, p_{8}\right\}$. We can calculate this for the three cases corresponding to $3 \geq\langle\mathcal{A}\rangle>1,1 \geq\langle\mathcal{A}\rangle>-1$, and $-1 \geq\langle\mathcal{A}\rangle \geq-3$, as follows. When $\langle\mathcal{A}\rangle$ lies between 3 and 1 , the measured value for $\mathcal{A}$ can take only 3 or 1 but not -1 or -3 to attain the minimum variance of its distribution. The minimization of $\left\langle(\Delta \mathcal{A})^{2}\right\rangle$ thus leads to the distribution $\left\{f, p_{\beta}, p_{\alpha}, p_{8}\right\}$ to be $\left\{\frac{\langle\mathcal{A}\rangle-1}{2}, \frac{3-\langle\mathcal{A}\rangle}{2}, 0,0\right\}$. Similarly, for $1 \geq\langle\mathcal{A}\rangle>-1$ case, the distribution is calculated to be $\left\{0, \frac{1+\langle\mathcal{A}\rangle}{2}, \frac{1-\langle\mathcal{A}\rangle}{2}, 0\right\}$, and for $-1 \geq\langle\mathcal{A}\rangle \geq-3$ case to be $\left\{0,0, \frac{3+\langle\mathcal{A}\rangle}{2}, \frac{-1-\langle\mathcal{A}\rangle}{2}\right\}$. The derived fidelity in Case 3, by the minimum variance principle, is then summarized as

$$
f=\left\{\begin{array}{cl}
\frac{\langle\mathcal{A}\rangle-1}{2} & 3 \geq\langle\mathcal{A}\rangle>1 \\
0 & 1 \geq\langle\mathcal{A}\rangle \geq-3 .
\end{array}\right.
$$

It is easy to show that this is equal to the minimum of the fidelity values with the help of Eq. (16), where the parameters are set to be $M=3$ and $r_{2}=1$. It is thus concluded that, for Case 3 , the derived fidelity to $\left|\Phi_{3}\right\rangle$ by the minimum variance principle leads to the minimum of the possible fidelity values allowed by the expectation value.

We generalize the argument for $n$-partite system. We consider $\mathcal{A}=\alpha_{1} Q_{1}+\alpha_{2} Q_{2}+\cdots+\alpha_{m} Q_{m} \in \mathcal{C}_{n}$. Suppose that an expectation value $\langle\mathcal{A}\rangle$ is given. In the following we calculate the fidelity $f$ from the minimum variance principle in this case. We write the eigenvalues of $\mathcal{A}$ as $M, r_{2}, r_{3}, \cdots$, and $r_{s}$, where $M>r_{2}>r_{3}>\cdots>r_{s} \geq$ $-M$. The probabilities for observing these eigenvalues, $M, r_{2}, r_{3}, \cdots$, and $r_{s}$ are denoted as $f, q_{2}, q_{3}, \cdots$, and $q_{s}$, respectively. Similarly to the discussion as to Case 3 for tripartite system, if $\langle\mathcal{A}\rangle$ lies between $M$ and $r_{2}$, the minimization of $\left\langle(\Delta \mathcal{A})^{2}\right\rangle$ leads to $\sum_{i=3}^{s} q_{i}=0$, which means $\left\{f, q_{2}, q_{3}, \cdots, q_{s}\right\}=\left\{\frac{\langle\mathcal{A}\rangle-r_{2}}{M-r_{2}}, \frac{M-\langle\mathcal{A}\rangle}{M-r_{2}}, 0, \cdots, 0\right\}$. The derived fidelity is then summarized as

$$
f=\left\{\begin{array}{cc}
\frac{\langle\mathcal{A}\rangle-r_{2}}{M-r_{2}} & M \geq\langle\mathcal{A}\rangle>r_{2} \\
0 & r_{2} \geq\langle\mathcal{A}\rangle \geq r_{s}
\end{array}\right.
$$

Eq. (24) is a special case of Eq. (25) where the parameters are set to be $M=3, r_{2}=1$ and $r_{s}=-3$. We can see that Eq. (25) gives the minimum fidelity with the help of Eq. (16) 17]. Hence we have the following result:

Proposition 3: When $\langle\mathcal{A}\rangle:=\operatorname{Tr}[\rho \mathcal{A}]$ is given, where $\mathcal{A} \in \mathcal{C}_{n}$, the derived fidelity to $\left|\Phi_{n}\right\rangle$ from the minimum variance principle is the minimum of the possible fidelity values allowed by the expectation value.

\section{CONCLUSION}

In conclusion, we have analyzed the possible fidelity values that are compatible with an expectation value of a single operator as experimental data. We have defined the desired maximally entangled state and formulated one class that is related to a decomposition of the projector onto the desired state. We have made use of the commutative group theory to formulate a class of observables. When an expectation value of an operator that belongs to the class is given, we can derive an inequality that gives a lower bound and an upper bound of the fidelity values that are compatible with the expectation value. With the help of the inequality, we have analyzed the experimental data by Pan et al. 4]. The data ensure the observed state does not belong to the biseparable class and has genuinely tripartite entanglement. Finally, we have also analyzed the calculated fidelity from the minimum variance principle.

\section{Acknowledgments}

The authors are very grateful to S. Takagi for calling our attention to the present problem, and also A. Miranowicz for his intensive reading of the manuscript. This work was partly supported by the Grant-in-Aid for Scientific Research (B) (Grant No. 12440111) by Japan Society of the Promotion of Science.
[1] D. M. Greenberger, M. A. Horne, and A. Zeilinger, in Bell's Theorem, Quantum Theory and Conceptions of the Universe, edited by M. Kafatos (Kluwer Academics, Dordrecht, The Netherlands 1989), pp. 69-72; see also N. D. Mermin, Phys. Rev. Lett. 65, 1838 (1990).

[2] See, for example, M. Hillery et al., Phys. Rev. A 59, 1829 (1999).

[3] D. Bouwmeester et al., Phys. Rev. Lett. 82, 1345 (1999).

[4] J. -W. Pan, D. Bouwmeester, M. Daniell, H. Weinfurter,
A. Zeilinger, Nature (London) 403, 515 (2000).

[5] C. A. Sackett et al., Nature (London) 404, 256 (2000); A. Rauschenbeutel et al., Science 288, 2024 (2000); J. -W. Pan et al., Phys. Rev. Lett. 86, 4435 (2001).

[6] See R. Jozsa, J. Mod. Opt. 41, 2315 (1994).

[7] A. Peres, Phys. Rev. Lett. 77, 1413 (1996); C. H. Bennett et al., Phys. Rev. Lett. 76, 722 (1996); M. Murao et al., Phys. Rev. A 57, R4075 (1998); W. Dür et al., Phys. Rev. Lett. 83, 3562 (1999); W. Dür et al., Phys. Rev. A 
61, $042314(2000)$.

[8] A. Acín et al., Phys. Rev. Lett. 87, 040401 (2001).

[9] The states beyond the biseparable class are further classified into the GHZ class and the W class. The GHZ witness is given by

$$
\mathcal{W}_{\mathrm{GHZ}}=\frac{3}{4} \mathbb{1}-P_{\mathrm{GHZ}} .
$$

When $\operatorname{Tr}\left[\mathcal{W}_{\mathrm{GHZ}} \rho\right]<0$, the state $\rho$ belongs to the GHZ class and not to the $W$ class. See 8$]$.

[10] E. T. Jaynes, Phys. Rev. 106, 620 (1957) ; ibid. 108, 171 (1957).

[11] R. Horodecki et al., Phys. Rev. A 59, 1799 (1999).

[12] A. K. Rajagopal, Phys. Rev. A 60, 4338 (1999).

[13] S. Abe et al., Phys. Rev. A 60, 3461 (1999); A. Rigo et al., Phys. Lett. A 270, 1 (2000); T. A. Brun et al.,
Phys. Rev. A 63, 042309 (2001); J. Batle et al., e-print quant-ph/0107023

[14] See V. Bužek et al., J. Mod. Opt. 44, 2607 (1997).

[15] See M. Seevinck and J. Uffink, Phys. Rev. A 65, 012107 (2002).

[16] This is due to the following fact: The measured value for $\mathcal{A}=\mathcal{O}_{x y y}+\mathcal{O}_{y x y}+\mathcal{O}_{z z I}$ can take only two values 3 and -1 , where both of them are degenerate eigenvalues.

[17] Incidentally, the maximization of $\left\langle(\Delta \mathcal{A})^{2}\right\rangle$ leads the distribution $\left\{f, \quad q_{2}, \quad q_{3}, \cdots, q_{s}\right\}$, to be $\left\{\frac{\langle\mathcal{A}\rangle-r_{s}}{M-r_{s}}, 0, \cdots, 0, \frac{M-\langle\mathcal{A}\rangle}{M-r_{s}}\right\}$. We can see that this gives the maximum fidelity with the help of Eq. (16). 